\title{
Traduire
}

Ine autre perspective sur I t tadaduction

Revue française de la traduction

$242 \mid 2020$

Passons au vert

\section{L'écotraduction, ou le traducteur en transition}

Optimiser son environnement de travail pour réduire son empreinte écologique

\section{Séverine George et Marie-Laure Faurite}

\section{(2) OpenEdition}

1 Journals

Édition électronique

URL : http://journals.openedition.org/traduire/1971

DOI : 10.4000/traduire.1971

ISSN : 2272-9992

Éditeur

Société française des traducteurs

Édition imprimée

Date de publication : 30 juin 2020

Pagination : 6-22

ISSN : 0395-773X

\section{Référence électronique}

Séverine George et Marie-Laure Faurite, «L'écotraduction, ou le traducteur en transition », Traduire [En ligne], 242 | 2020, mis en ligne le 15 juillet 2020, consulté le 20 décembre 2020. URL : http:// journals.openedition.org/traduire/1971; DOI : https://doi.org/10.4000/traduire.1971 


\section{L'écotraduction, ou le traducteur en transition}

Optimiser son environnement de travail pour réduire son empreinte écologique

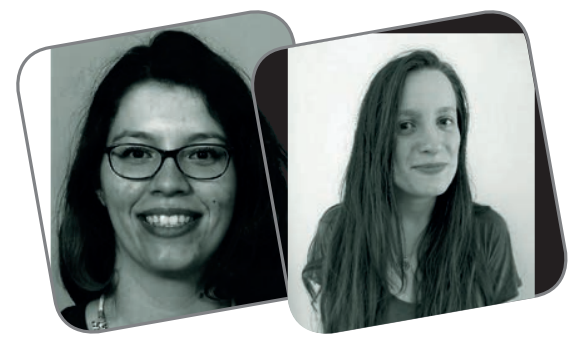

Séverine George, Marie-Laure Faurite

"The challenge now is to bring translation to the heart of the dialogue about the future of our shared planet."

Michael Cronin

La protection de l'environnement est un sujet de plus en plus présent que nous ne pouvons pas ignorer. Que ce soient les scientifiques (rapports du Giec, de I'IPBES, etc.), les politiques (accords de Paris, décisions liées aux transitions écologiques), les entreprises (rapports RSE) ou même les particuliers (conscience environnementale grandissante, écoanxiété, etc.), tous les acteurs de la société semblent s'engager dans ce sens et adopter de nouvelles pratiques écoresponsables.

Chaque jour, nous devons faire face à de nouvelles informations et injonctions qui se rapportent de près ou de loin à l'environnement. Difficile parfois de s'y retrouver entre les articles alarmistes et culpabilisants, prônant une écologie extrémiste, et les publications plus optimistes, voire parfois climatosceptiques. 
Nous, traducteurs et traductrices, avons aussi un impact environnemental non négligeable puisque, à l'heure où nous écrivons, l'informatique représente $4 \%$ des émissions mondiales de gaz à effet de serre (Ademe, 2019). Notre métier étant devenu dépendant des nouvelles technologies, il paraît crucial de tenter de réduire notre empreinte.

Dans ce contexte, quelles sont les actions que le traducteur peut, à son tour, mettre en place pour agir à son échelle?

Nous avons imaginé le personnage de l'écotraducteur: l'archétype du traducteur écoconscient qui aurait réfléchi à diverses démarches pour minimiser l'impact carbone de son activité. Cet article présente donc les «écogestes»que ce traducteur un peu particulier met en place. II regroupe un ensemble d'explications et de conseils simples et applicables au quotidien pour vous inviter à trouver vos propres postures écologiques et vous encourager à commencer, à votre tour, à écotraduire.

\section{L'environnement de travail, une première prise de conscience}

Le télétravail, certainement la façon la plus écologique d'exercer une activité professionnelle, est particulièrement adapté à la traduction. II évite les déplacements quotidiens et l'utilisation d'un local dédié.

Comment l'écotraducteur repense-t-il son lien avec ses équipements de bureau et prend-il conscience de l'impact de chacune de ses actions?

Ces premiers conseils restent très généraux et sont applicables à d'autres professions.

\section{Réparer au lieu de racheter}

De manière générale, l'écotraducteur ne cherche pas à remplacer fréquemment son équipement informatique par les produits les plus performants du marché. II se renseigne au contraire sur les possibilités d'acheter d'occasion. Si l'un de ses outils de travail s'abîme, il essaie de le réparer, en consultant des tutoriels ou en se rendant dans des "Repair Cafés», ces locaux où des bénévoles aident à remettre les 
objets en état (Repair Café, 2020). Quand la réparation se révèle impossible, il recycle alors correctement l'équipement.

\section{Régler son chauffage et sa climatisation}

Selon l'Agence de l'environnement et de maîtrise de l'énergie (Ademe), le chauffage représente $50 \%$ des dépenses énergétiques des bureaux (Ademe, 2019). L'écotraducteur utilise raisonnablement le chauffage et la climatisation de son lieu de travail. Il adopte des pratiques adaptées à chaque saison: en été, il ferme les volets et a recours à un ventilateur ou se rend dans des espaces de travail partagés climatisés, tandis qu'il se couvre davantage et régule son chauffage en hiver. Dans son guide Se chauffer mieux et moins cher, l'Ademe recommande une température comprise entre 19 et $21^{\circ} \mathrm{C}$ la journée et $17{ }^{\circ} \mathrm{C}$ la nuit (Ademe, 2018).

\section{Penser écologie et ergonomie}

L'écotraducteur place son bureau en fonction de la luminosité ambiante. Ce conseil se veut à la fois écologique et ergonomique. L'écotraducteur est sensibilisé aux impacts des différentes technologies en matière d'ampoules (consommation, durée de vie, éclairage, etc.). II les recycle convenablement en les apportant en déchetterie ou bien en bacs de recyclage disponibles dans les magasins.

\section{Changer de fournisseur d'électricité}

En 2017, la production d'électricité a généré 27,9 millions de tonnes de $\mathrm{CO}_{2}$ en France (Le Guide de l'électricité verte, 2018). L'écotraducteur fait également le choix d'être un «consommacteur» en optant pour un fournisseur d'électricité plus responsable. Le Guide de l'électricité verte propose des fournisseurs alternatifs, en étudiant les parts d'énergies renouvelables pour chacun.

\section{Choisir une banque en accord avec ses principes}

Le choix de la banque n'est pas anodin. L'écotraducteur a conscience que l'empreinte carbone des banques varie 
en fonction du type d'activités qu'elles financent. En effet, l'argent placé sur les comptes bancaires est réinjecté dans le circuit économique pour financer des projets, parfois très polluants. L'association les Amis de la Terre propose un guide qui offre un apercu des alternatives aux banques traditionnelles.

\section{Le classement des banques}

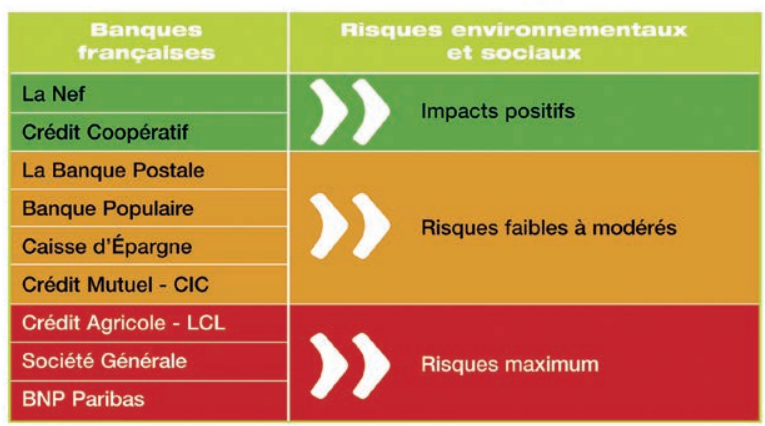

Classement des banques en fonction de leur impact sur le climat (Les Amis de la Terre, 2014).

\section{Imprimer sobrement}

L'écotraducteur a pour principe de réduire ses impressions au maximum, d'utiliser du papier recyclé, ou, à défaut, du papier labellisé (par exemple FSC ou PEFC). Il est recommandé d'imprimer recto verso ou de se servir des versos vierges de feuilles comme brovillon.

L'écotraducteur fait remplir ses cartouches d'encre dans des magasins dédiés. Cette pratique permet d'éviter les déchets produits par les cartouches jetables, tout en réalisant d'importantes économies. Plus de 300 millions de cartouches d'encre toner laser et jet d'encre sont jetées chaque année (RecyclingTimes, 2013).

Il existe en outre des astuces pour utiliser moins d'encre et ainsi faire durer les cartouches plus longtemps. En effet, certaines polices d'impression sont moins gourmandes en encre, par exemple Garamond, souvent préinstallée sur les logiciels. Une étude de Grenoble INP-Pagora analyse différentes polices en fonction de leur confort de lecture et de leur consommation d'encre (Ajas, 2017). 
À titre d'exemple, la police Garamond permet d'économiser $36 \%$ d'encre comparée à Arial', soit une économie de 2 centimes d'euro par feuille imprimée 2 .

Le style de police n'est pas non plus anodin. En effet, d'après cette étude, pour 200 feuilles imprimées en police Arial, taille 10 en gras, la masse d'encre s'élève à 18,6 g tandis qu'en mode normal, elle ne dépasse pas 18,2 $\mathrm{g}$. La différence est encore plus flagrante en niveau de gris à $85 \%$, mode généralement proposé par les imprimantes domestiques, puisque la masse d'encre se limite alors à $11 \mathrm{~g}$ par feuille. Quelle que soit la police utilisée, l'écotraducteur adopte donc les écogestes suivants: il privilégie le style normal et imprime, lorsqu'il le peut, en $85 \%$ de noir.

\section{Repenser son matériel de papeterie}

L'écotraducteur utilise des stylos rechargeables et non pas jetables. De même, il peut remplacer ses surligneurs par des crayons de couleur. Il existe de nombreuses marques de crayons écologiques, faits à partir de papier recyclé, compostables, ou même que l'on peut planter.

\section{Trier les déchets, même à son bureau}

L'écotraducteur installe une poubelle de recyclage à proximité de son bureau pour faciliter le tri des déchets. Il tient 
compte des consignes et des possibilités de collecte de sa commune.

\section{Réfléchir à l'impact de chaque achat}

Écotraduire consiste également à réfléchir à ses achats, tant en amont qu'en aval, et à la fin de vie des produits. L'écotraducteur prête une attention particulière aux déchets d'équipements électriques et électroniques (DEEE) qui contiennent souvent des substances et composants dangereux, mais qui offrent aussi un fort potentiel de recyclage (métaux, verre et plastiques) (Ministère de la Transition écologique et solidaire, 2020). En 2009, ils représentaient près de 40 millions de tonnes (Flipo, 2014).

En ce qui concerne la bureautique, il est fréquent que l'achat neuf soit d'une part le plus pratique, et d'autre part le plus rassurant. Si l'Ademe estime que "faire durer nos équipements numériques constitue le geste le plus efficace pour diminuer leurs impacts» (Ademe, 2019), avoir une garantie de la qualité de son téléphone portable ou ordinateur reste un avantage non négligeable, sans compter qu'un appareil récent sera probablement plus performant.

\section{Opter pour du neuf durable}

Il est alors conseillé de se renseigner sur les alternatives aux marques polluantes. Les options modulables, réparables et plus éthiques (au niveau des matériaux utilisés et de la rémunération des travailleurs) sont préférables à des achats qui encouragent un commerce fondé sur l'obsolescence programmée. Des téléphones modulables (Fairphone, par exemple) sont déjà disponibles sur le marché. II existe également un projet de conception d'ordinateurs modulables français: I'Open Computer (Makery, 2018).

Lorsqu'il achète du neuf, l'écotraducteur se renseigne sur les labels environnementaux relatifs aux appareils numériques (Bordage, 2007). Il étudie aussi la différence de consommation entre un ordinateur portable et un fixe. En effet, selon l'Ademe et Greenlt, un ordinateur portable consommerait 30 à $100 \mathrm{kWh} / \mathrm{an}$ contre 120 à $250 \mathrm{kWh} / \mathrm{an}$ pour un ordinateur fixe (Ademe, 2019). Le site MSI propose 
de calculer la consommation électrique de son ordinateur (MSI, 2020).

\section{Éteindre les appareils}

Il est préférable d'éteindre les appareils pour éviter la consommation d'électricité induite par le mode veille. Par exemple, l'Ademe estime qu'un ordinateur en veille utilise entre 20 et $40 \%$ de l'équivalent de sa consommation en marche (Ademe, 2017). L'écotraducteur adopte des multiprises pour ses écrans qui, bien qu'éteints, continuent à consommer (consommation d'énergie "fantôme»). C'est d'ailleurs ce que recommande Béa Johnson, l'auteure du livre Zéro déchet (Johnson, 2013).

L'écotraducteur privilégie le mode veille pour de courtes absences.

\section{Réduire la consommation des appareils mobiles}

L'écotraducteur désactive les fonctions GPS, Wi-Fi et Bluetooth lorsqu'elles ne sont pas utiles. Quand il n'utilise pas son téléphone, il privilégie le mode "avion» ou l'éteint. Le mode «économie d'énergie» disponible sur la plupart des smartphones permet aussi d'en réduire la consommation.

\section{Se former tout en respectant l'environnement}

Le credo de l'écotraducteur: refuser de créer de futurs déchets. Lorsqu'il visite des salons, qu'il assiste à des formations ou à des conférences, il est souvent accueilli avec des blocs de feuilles, des stylos, tasses, boîtes, sacs et autres cadeaux à l'effigie de l'entreprise, de l'association ou de l'université organisatrice. L'écotraducteur part en formation ou en conférence muni de son propre matériel.

De plus en plus d'initiatives vont dans le sens du zéro déchet lors de ces événements. La SFT elle-même l'intègre petit à petit pour ses rencontres.

Si l'événement n'a pas lieu près de chez lui, l'écotraducteur évite de prendre l'avion. Il privilégie toujours le train ou le covoiturage pour ses déplacements professionnels. 


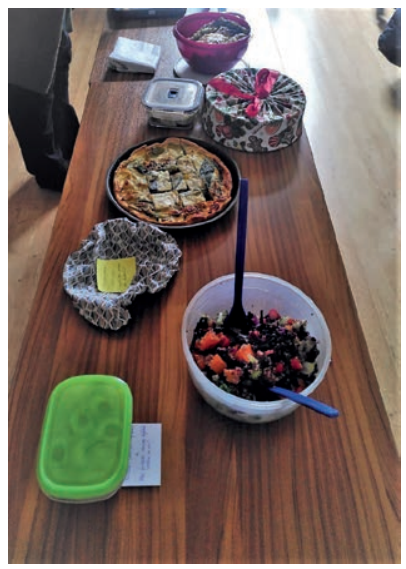

Repas zéro déchet lors de la réunion de la délégation SFT Rhône-Alpes en février 2020 à Grenoble.

\section{Des pratiques écoresponsables pour mieux gérer ses projets}

Le traducteur consacre une grande partie de son temps à réaliser des recherches en amont, à consulter des sites de référence, voire à établir un glossaire. Toute cette phase d'analyse a un coût écologique. Comment l'écotraducteur réduit-il l'empreinte carbone de ses recherches?

\section{Effectuer de bonnes recherches}

Selon l'Ademe, une bonne recherche doit être rapide, directe et précise (grâce à l'utilisation d'opérateurs booléens pour exclure des mots, trouver des groupes nominaux précis, sélectionner certaines URL, etc.). L'écotraducteur utilise les options "Précédent» et "Suivant» du navigateur au lieu de recharger des pages déjà visitées pour éviter de relancer des recherches inutiles.

Choisir avec soin les pages consultées est également primordial puisque chaque page visionnée sur ordinateur consomme en moyenne 466 mWh (Green Code Lab, 2014) ${ }^{3}$.

3. Sur la base d'un ordinateur disposant d'une alimentation de $42 \mathrm{~W}$ et d'une durée de consultation de $40 \mathrm{~s}$. 
L'écotraducteur prend l'habitude de fermer systématiquement les onglets qui ne lui servent plus. II existe également des extensions pour navigateurs qui ferment les onglets inactifs (Tab Suspender) (Vidal, 2019).

\section{Choisir son moteur de recherche}

Selon l'Ademe, un Français génère $9,9 \mathrm{~kg}$ de $\mathrm{CO}_{2}$ par an à raison d'une moyenne de 2,6 recherches effectuées par jour (Ademe, 2011). Le choix du moteur de recherche est une question épineuse. Il est préférable d'opter pour des alternatives écologiques au géant Google. Il existe des moteurs de recherche écologiques solidaires qui réduisent directement leur impact carbone (tels que Ecosia, Lilo ou Ecogine). Chaque recherche fait en effet appel à plusieurs centres de traitement des données (datacenters) qui sont sollicités à chaque étape de la recherche: l'accès au moteur de recherche, puis aux résultats, et enfin au site recherché. Dans la pratique, Google semble donner les résultats les plus pertinents dans une situation de recherche terminologique, comparé à plusieurs alternatives. Par conséquent, l'écotraducteur peut utiliser un moteur de recherche alternatif pour ses recherches privées et conserver Google pour ses recherches professionnelles. L'écotraducteur entre l'adresse exacte d'un site dans la barre d'adresse, ce qui permet d'éviter d'utiliser un moteur de recherche et ainsi de diviser par quatre les émissions de gaz à effet de serre.

\section{Choisir un navigateur}

Des extensions permettent de calculer l'empreinte écologique de la navigation, toujours dans le but d'atteindre la sobriété numérique (comme Carbonanalyser, créé par The Shift Project, un think tank qui milite pour proposer des alternatives à une économie reposant sur la contrainte carbone).

Tous les navigateurs ne consomment pas la même quantité d'énergie. Chrome consomme par exemple en moyenne $27 \mathrm{mWh}$ de plus que Firefox et Internet Explorer par page consultée (Green Code Lab, 2014). 
L'écotraducteur conserve les sites qu'il visite fréquemment en favoris, afin de minimiser le nombre de recherches effectuées. Cela s'applique tout particulièrement aux dictionnaires en ligne, sites de références, plateformes ou Dropbox des clients, réseaux sociaux, ainsi qu'aux sites des associations, syndicats ou agrégateurs de flux RSS.

Carbonalyser

Informations Mentions légales

$\cup$ Stopper l'analyse $\cup$ Ré-initialiser les données

Analyse en cours. Naviguez sur le web puis revenez ici pour les résultats.

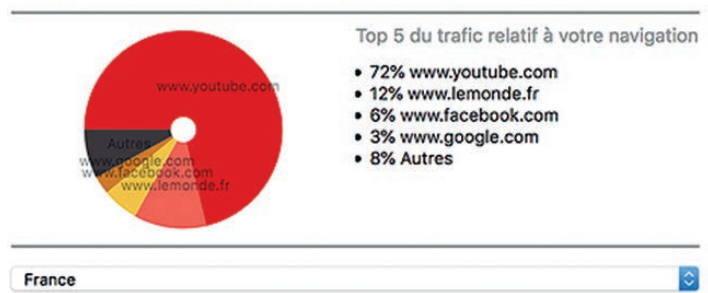

Ce n'est pas obligatoire mais cela rendra le résultat plus pertinent.

En 3 minutes de navigation, vous avez fait transiter 68 Mo de données. Cela a nécessité $0.16 \mathrm{kWh}$ d'électricité, soit $83 \mathrm{gCO} 2$
$C_{j}^{3}{ }_{\text {minutes }}^{3}$
$3 \underset{\mathrm{kWh}}{0.16}$
$\uparrow \downarrow$
(1) 83

Les émissions de $\mathrm{CO} 2$ liées à votre utilisation d'internet équivaut à :
ब 10
10
$\underset{0 \rightarrow \infty}{0.377} 0$

Analyse de consommation du navigateur sur 3 minutes d'utilisation normale (recherches terminologiques, gestion du site internet, réseaux sociaux professionnels).

\section{Apprendre à gérer le cache}

On entend souvent parler du cache du navigateur, mais de quoi s'agit-il? Jean-Michel Davault, auteur de l'article "Le coût énergétique d'indexation des données», définit le cache comme toute information déjà demandée qui est disponible sans devoir effectuer une nouvelle requête (Davault, 2012). Concernant sa gestion, deux écoles s'opposent: d'un côté, l'Ademe préconise de vider le cache fréquemment pour réduire l'espace de stockage local (Ademe, 2019); de l'autre, Jean-Michel Davault soutient que vider le cache va à l'encontre d'une optimisation énergétique, précisément parce 
que ce dernier permet de diminuer l'information demandée au serveur ${ }^{4}$. Le rapport final du projet Web Energy Archive du Green Code Lab conclut que la bonne pratique en matière de cache consiste à configurer les éléments à mettre en cache (Green Code Lab, 2014).

\section{La TAO, un outil pour écotraduire}

Toute action permettant d'éviter de recharger des pages sur internet peut être considérée comme une bonne pratique énergétique. En ce sens, l'utilisation de la TAO (et donc des mémoires et des bases terminologiques) pour les projets volumineux de clients réguliers paraît judicieuse. Une base terminologique déjà remplie dispense d'un nombre conséquent de recherches.

\section{Ne pas négliger les ressources papier}

En outre, même s'il reste à l'affût des nouveaux sites et applications à consulter, l'écotraducteur n'oublie pas les ressources papier existantes qui lui permettent de trouver des réponses sans forcément effectuer de recherche énergivore.

\section{Tendre vers une communication et un démarchage plus sobres}

Une partie de l'activité du traducteur indépendant est consacrée à la communication, que ce soit entre collègues ou avec les clients directs ou agences. Le numérique est, là aussi, incontournable. Comment l'écotraducteur peut-il être le plus sobre possible, tout en continuant à communiquer efficacement?

\section{Adopter un téléphone «double-SIM»}

Les smartphones ont un impact environnemental non négligeable (Ademe, 2019), ce dont l'écotraducteur a conscience.

4. Informations complémentaires obtenues lors d'un échange de messages avec JeanMichel Davault. 
De nombreux traducteurs souhaitent différencier leur numéro de mobile professionnel de leur numéro personnel. Vaut-il alors mieux opter pour deux téléphones ou bien un seul, avec une possibilité de mettre deux cartes SIM? Le rapport de The Shift Project propose d'augmenter la part de smartphones «pro-perso» (Ferrebœuf, 2018).

\section{Tendre vers l'écocommunication}

L'écotraducteur adopte certaines pratiques en matière de gestion de sa messagerie professionnelle.

En effet, envoyer un courriel accompagné d'une pièce jointe équivaut à laisser une ampoule allumée pendant 24 heures (Ademe, 2011). L'écotraducteur est donc adepte de l'écocommunication, c'est-à-dire qu'il évite les courriels superflus, tant au niveau de la réception que de l'envoi ${ }^{5}$.

\begin{tabular}{|c|c|c|c|c|c|}
\hline Indicateur & Unité & Méthode & $\begin{array}{l}\text { Envoi d'1 mail de } 1 \\
\text { Mo à } 1 \text { personne }\end{array}$ & $\begin{array}{l}\text { Envoi d'1 mail de } 1 \\
\text { Mo à } 10 \text { personnes }\end{array}$ & $\begin{array}{l}\text { Envoi d' } 1 \text { mail de } 1 \\
\text { Mo à } 100 \text { personnes }\end{array}$ \\
\hline $\begin{array}{l}\text { Potentiel de } \\
\text { changement climatique }\end{array}$ & géq $\mathrm{CO}_{2}$ & ReCiPe & 19 & 73 & 615 \\
\hline $\begin{array}{l}\text { Epuisement potentiel } \\
\text { des métaux }\end{array}$ & $g$ éq Fe & ReCiPe & 4,8 & 7,0 & 29 \\
\hline $\begin{array}{l}\text { Epuisement potentiel } \\
\text { des ressources fossiles }\end{array}$ & $\mathrm{g}$ éq pétrole & ReCiPe & 5,2 & 22 & 186 \\
\hline
\end{tabular}

Analyse du cycle de vie de l'envoi d'un courriel de 1 Mo (Ademe, 2011).

\section{Trier ses courriels}

L'écocommunication passe aussi par un meilleur tri des courriels, puisque les messages conservés continuent à faire tourner les serveurs de la messagerie. C'est ce que l'on appelle la «pollution dormante». L'écotraducteur met en place un système de classement par dossier pour optimiser son organisation et distinguer les pourriels des courriels.

5. L'équivalent $\mathrm{CO}_{2}$ a été créé pour mesurer le potentiel de réchauffement global des gaz à effet de serre, avec le $\mathrm{CO}_{2}$ comme référence.

L'analyse du cycle de vie permet d'évaluer les impacts environnementaux d'un produit ou service en quantifiant les flux (matières et énergie) entrants et sortants. La méthode $\mathrm{ReCiPe}$ analyse les flux recensés qui sont triés en catégories. Elle distingue les midpoints, 18 indicateurs qui quantifient les changements environnementaux (acidification terrestre, eutrophisation marine...) et les endpoints, qui mesurent les dommages sur les écosystèmes, la santé humaine et les ressources. 
Il installe également des filtres antispam dans sa messagerie. Des sites et applications permettent à l'écotraducteur de nettoyer automatiquement sa messagerie et de se désinscrire des lettres d'information (Cleanfox).

Par ailleurs, l'écotraducteur se renseigne sur des moyens de communication plus efficaces.

\section{Privilégier les sites de dépôt temporaire pour les fichiers}

En ce qui concerne l'envoi de documents, notamment à plusieurs destinataires, l'écotraducteur privilégie des sites de dépôt temporaire, sur lesquels les données sont effacées au bout de quelques jours et non stockées indéfiniment sur des serveurs. Certaines plateformes se présentent comme des alternatives écologiques, par exemple Swisstransfer, la plateforme d'Infomaniak (Infomaniak, 2020), qui déclare compenser l'intégralité de ses émissions de $\mathrm{CO}_{2}$ grâce à ses centres de traitement des données moins gourmands, des techniques de régulation de l'air plus efficaces, des équipements plus durables et des solutions de recyclage adaptées. Pour les plus gros fichiers, l'Ademe recommande d'utiliser une clé USB.

\section{Mieux gérer les pièces jointes}

Si des fichiers doivent impérativement être envoyés par courriel, l'écotraducteur supprime les pièces jointes après les avoir enregistrées sur son ordinateur. Les fichiers compressés ou en basse résolution sont également à privilégier. Cela vaut notamment pour les signatures professionnelles contenant des images et envoyées à chaque échange.

\section{Opter pour un hébergeur de messagerie écoresponsable}

L'écotraducteur s'intéresse également aux hébergeurs alternatifs. En effet, ces derniers communiquent de façon transparente sur la localisation des centres de traitement des données et leur refroidissement, utilisent des sources d'énergie alternatives ou compensent l'empreinte carbone de l'activité induite. Parmi les principales alternatives figurent Ecomail (qui reverse $50 \%$ des recettes à des associations environnementales), Newmanity (qui ne commercialise pas 
les données personnelles et participe à la reforestation), ou encore Infomaniak (dont les serveurs sont en Suisse et qui a signé une charte écologique ambitieuse).

\section{Se tourner vers une solution de synchronisation plus durable}

Les solutions de synchronisation permettent aux traducteurs d'accéder à un ensemble de documents sur n'importe quel appareil et facilitent donc les échanges entre collègues et avec les clients. Au premier abord, sobriété énergétique et stockage en nuage (cloud) semblent antithétiques. Cependant, ces solutions sont parfois indispensables et ont l'avantage d'éviter des envois de courriels et de piècesjointes, le recours à un disque dur, les impressions, etc. L'utilisation d'une plateforme synchronisée sur un serveur partagé permet de diminuer de $81 \%$ les émissions de gaz à effet de serre, comparé à un envoi par courriel (Ferrebœuf, 2018) ${ }^{6}$.

L'écotraducteur choisit sa plateforme sciemment en évaluant précisément ses besoins en matière d'espace de stockage. Il se tourne vers des alternatives sécurisées et responsables en se renseignant sur l'éthique des produits (revente des données des utilisateurs, utilisation exclusive d'énergie renouvelable locale, compensation des émissions de $\mathrm{CO}_{2}$, refroidissement des serveurs, emplacement géographique, etc.). Cependant, peu d'études ont été menées sur la consommation de l'informatique en nuage. L'indicateur d'efficacité énergétique ${ }^{7}$ est la métrique principale permettant d'évaluer la consommation des centres de traitement des données. Les plateformes qui proposent des alternatives écologiques sont encore rares. II existe toutefois le kDrive d'Infomaniak, cité plus haut, ou hubiC d'OVHcloud, un hébergeur français sensibilisé depuis des années aux techniques de refroidissement des centres (OVHcloud, 2020).

L'écotraducteur adopte par ailleurs des bonnes pratiques en triant les données qu'il conserve en ligne et en archivant ses

6. Calcul basé sur le partage de quatre versions d'un document de 1 mégaoctet par cina collaborateurs.

7. Le Power Usage Effectiveness (PUE) indique le ratio entre l'énergie consommée par le centre d'exploitation (refroidissement, climatisation...) et celle consommée par les équipements (réseau, serveurs, stockage). Plus le résultat est proche de 1, plus la consommation énergétique est basse. 
anciens documents en local. Il évite autant que possible de stocker des fichiers lourds (images, vidéos, etc.) sur le serveur.

\section{Écoconcevoir son site}

Le site d'un traducteur est un outil mercatique au même titre que sa page Linkedln ou que sa carte de visite.

Les sites peuvent, eux aussi, être écoconçus. Cela consiste à concevoir un site d'une qualité sensiblement équivalente à celle d'un site classique, en mobilisant toutefois bien moins de ressources (Réseau du futur, 2019). Des plateformes proposent d'écoconcevoir les sites (par exemple GreenlT) et il existe un guide qui présente 115 bonnes pratiques d'écoconception (Bordage, 2015), notamment: optimiser les images pour alléger les pages du site, choisir un hébergeur «vert», favoriser un design épuré et des formats adaptés au web, limiter le recours aux extensions (plug-ins) et l'utilisation de Flash.

\section{Conclusion}

Les conseils pour amorcer des transitions afin de réduire autant que possible son empreinte carbone sont nombreux. Quoi qu'il en soit, une traduction entièrement écologique et neutre serait une utopie, justement parce que la mise en place de toutes ces pratiques demande beaucoup de recherches préalables, que certaines pratiques écologiques représentent un investissement conséquent et qu'il est difficile d'être irréprochable.

Il ne s'agit pas ici de culpabiliser, mais au contraire, de permettre une prise de conscience du poids de la pollution invisible à laquelle les traducteurs contribuent quotidiennement et de proposer des pistes pour ceux qui aimeraient tendre vers davantage de sobriété. Petit à petit, cette prise de conscience et ces actions individuelles constitueront, à grande échelle, un changement sociétal. Nul besoin d'être parfait, donc, pour écotraduire. Il suffit d'être sensible à l'impact de son activité et d'essayer, peu à peu, de le minimiser.

Cet article n'aborde pas l'aspect du contenu des projets, qui peut parfois aller à l'encontre des principes du traducteur. En effet, il appartient à chacun de remettre en question 
la nature des projets qu'il reçoit. Privilégier des clients plus respectueux de l'environnement peut également être une posture très intéressante pour les écotraducteurs.

Un grand merci à tous les professionnels, collègues et proches qui nous ont conseillées lors de la rédaction de cet article.

contact@ecotraduire.com

Séverine George est traductrice (anglais et espagnol vers le français) et a suivi la formation de I'université Grenoble Alpes. Elle est spécialisée dans le domaine de l'environnement, particulièrement dans la biodiversité et la gestion des déchets, pour faire concorder son engagement personnel avec son activité professionnelle.

Marie-Laure Faurite est traductrice (anglais et allemand vers le français) diplômée de l'université Grenoble Alpes. Passionnée par les thématiques de l'environnement, des énergies et du traitement des déchets, elle est spécialisée dans le domaine de la traduction environnementale (RSE, documents scientifiques, etc.)

SOURCES CITÉES

Ademe, Analyse comparée des impacts environnementaux de la communication par voie électronique, Paris, Bio Intelligence Service, 2011, http://bit.ly/39lQT5n, consulté le $20 / 05 / 2020$.

Ademe, Écoresponsable au bureau, Angers (France), Ademe, coll. "Au quotidien", septembre 2019 .

Ademe, La face cachée du numérique, Angers (France), Ademe, coll. "Au quotidien », novembre 2019 .

Ademe, Le site de l'éco-communication, France, Ademe, 2016, http://bit.ly/2Tkn6nO, consulté le 20/05/2020.

Ademe, Les équipements électriques, France, Ademe, 2017, http://bit.ly/3aoEaPb, consulté le 20/05/2020.

Ademe, Les impacts du smartphone, Angers (France), Ademe, coll. «Mes achats», décembre 2019. 
Ademe, Se chauffer mieux et moins cher, Angers (France), Ademe, coll. «Chauffage/ eau chaude sanitaire", septembre 2018.

AJAS Anne-Sophie, «Quelle police consomme le moins d'encre?», Grenoble, Cerig, 2017, http://bit.ly/2vFdHOC, consulté le 20/05/2020.

BORDAGE Frédéric, Écoconception web. Les 115 bonnes pratiques, Paris, Eyrolles, 2015.

BORDAGE Frédéric, Les éco-labels, France, GreenIT.fr, 2008, https://bit.ly/3cPbQ9P, consulté le 08/61/2020.

CRONIN Michael, Eco-translation: Translation and Ecology at the Age of the Anthropocene, Londres et New York, Routledge, New Perspectives in Translation and Interpreting Studies, 2017.

DAVAULT Jean-Michel, «Le coût énergétique d'indexation des données », France, Green Code Lab, 2012, http://bit.ly/39kO9Fa, consulté le 20/05/2020.

FERREBCEF Hugues (dir.), LEAN ICT-Pour une sobriété numérique, France, The Shift Project, 2018.

FLIPO Fabrice, «Expansion des technologies de l'information et de la communication: vers l'abîme?", in Mouvements, n $^{\circ} 79,2014$, p. 115-121.

Green Code Lab, Web Energy Archive (WEA), France, Green Code Lab, 2014, http:// bit.ly/32Vql8E, consulté le 20/05/2020.

Infomaniak, Hébergeur écologique, France, Infomaniak, http://bit.ly/2Tz1Xp6, consulté le 20/05/2020.

JOHNSON Béatrice, Zéro déchet, Paris, Les Arènes, 2013.

Le Guide de l'électricité verte, Votre fournisseur d'électricité est-il vraiment vert?, France, Greenpeace, 2018, http://bit.ly/2TyLEbG, consulté le 20/05/2020.

Les Amis de la Terre, Climat: Comment choisir ma banque?, Montreuil (France), Les Amis de la Terre, 2014.

Makery, Open Computer ou la possibilité d'un portable modulaire libre, Makery, 2018, http://bit.ly/2PL5uzA, consulté le 20/05/2020.

Ministère de la Transition écologique et solidaire, Déchets d'équipements électriques et électroniques, France, ministère, 2020, http://bit.ly/2IknsVb, consulté le $20 / 05 / 2020$

MSI, Calculer l'alimentation nécessaire, France, MSI, http://bit.ly/38lL9qT, consulté le $20 / 05 / 2020$.

OVHcloud, OVH.COM, expert en datacentre, France, OVHcloud, https://bit. ly/2UJaPdk, consulté le 20/05/2020.

Repair Café, À propos, France, Repair Café, http://bit.ly/2VKFaJs, consulté le 20/05/2020.

RecyclingTimes, «The Lists», RecyclingTimes, Chine, $\mathrm{n}^{\circ} 42$, 2013, p. 70.

Réseaux du futur, Note $n^{\circ} 5$ : l'empreinte carbone du numérique, France, Arcep, 2019.

VIDAL Julien, Ça va changer avec vous!, Paris, Éditions First, 2019. 